\title{
The Yarkovsky thermal force on small asteroids and their fragments
}

\section{Choosing the right albedo}

\author{
D. Vokrouhlický ${ }^{1}$ and W. F. Bottke Jr. ${ }^{2}$ \\ 1 Institute of Astronomy, Charles University, V Holešovičkách 2, 18000 Prague 8, Czech Republic \\ 2 Southwest Research Institute, 1050 Walnut St, Suite 426, Boulder, CO 80302, USA \\ e-mail: bottke@boulder.swri.edu
}

Received 13 February 2001 / Accepted 12 March 2001

\begin{abstract}
Thermal (Yarkovsky) forces acting on small bodies in the solar system have been shown to play an important role in the the delivery of meteoroids and $\mathrm{km}$-sized asteroids to Earth. So far, several aspects of the Yarkovsky force have been studied in some detail, including non-linear effects of the heat diffusion problem, nonsphericity and the layered-structure of the objects. In this paper, we address the concept of optical absorptivity. We find that the best formulation of the Yarkovsky effect uses the first two moment-integrals of an object's hemispheric albedo. A useful approximation of these albedo parameters is an object's Bond albedo.
\end{abstract}

Key words. celestial mechanics - minor planets, asteroids - meteoroids

\section{Introduction}

The Yarkovsky effect is a radiation recoil force causing objects to undergo semimajor axis drift according to their spin, orbit, and material properties (Rubincam 1995, 1998; Farinella et al. 1998; Vokrouhlický 1999). To accurately evaluate its importance in the dynamical evolution of small solar system bodies, we require more precise observations and improved theoretical models. As far as these theoretical models are concerned, we note that the principal step forward has been performed by Rubincam (1995), who extended the classical (rotationfrequency-dependent) formulation by the mean-motion variant (the so-called seasonal effect). Later, the solution of the Yarkovsky force has been extended for the non-spherical bodies (Vokrouhlický 1998b), objects with layered-structure (Vokrouhlický \& Brož 1999) and a degree by which the classical and seasonal variants of the Yarkovsky effects are mixed (Vokrouhlický 1999). At the same time, non-linearized models have been developed in both the large-body (Vokrouhlický \& Farinella 1998) and

Send offprint requests to: D. Vokrouhlický, e-mail: vokrouhl@mbox.cesnet.cz general approximations (Vokrouhlický \& Farinella 1999; Spitale \& Greenberg 2001).

Even though progress has been made in many areas, theoretical models of the Yarkovsky effect still need to be improved. Specifically, we believe that some attention should be paid to the selection of the appropriate "albedo" for an object evolving under Yarkovsky forces. Previous works have used several different types of albedos. For example, Rubincam $(1995,1998)$ refers to a single albedo value $A$ in his models, but usually considers $A=0$ in applications (at best, the Bond albedo has been substituted for the $A$-parameter, e.g. in Bottke et al. 2000). Vokrouhlický and Farinella, in their series of papers, also refer to a single value of the albedo parameter, assuming Lambert diffusion of the sunlight on the surface of the body. Similarly, none of the non-linearized theories of the Yarkovsky effect have examined the concept of surface albedo in detail.

To clarify the situation, it is useful to describe the differences between the various albedos used above. Two general types of albedo can be found in the literature, normal albedo and Bond albedo. The former, also called normal reflectance or geometric albedo, is a measure of a surface's relative brightness when illuminated and 
observed vertically. It derives from the hemispheric albedo, defined below, at zero zenith distance. The normal albedo is commonly used to determine the surface composition of asteroids. For reference, the normal albedo of (4) Vesta is nearly 0.35 , while the surface of (1) Ceres is 0.09 , not much brighter than charcoal. This parameter is used, along with diameter and distance, to determine the brightness of an object. Bond (sometimes spherical) albedo is defined as the fraction of the total incident solar radiation reflected by an object back to space. Hence, Bond albedo measures an object's energy balance. Working with bolometric quantities, the Bond albedo is frequently calculated by multiplying the normal albedo by an estimated phase integral (Lebofsky \& Spencer 1989). More precise computations of the Bond albedo for some asteroids can be found in the literature (e.g., 243 Ida; Helfenstein et al. 1996).

In this note, we aim to clarify the concept of albedo parameter(s) within the context of the Yarkovsky force theory. The reference papers, as far as the notation is concerned, are those by Vokrouhlický (1998a, 1999).

\section{Theory}

Consider a spherical body of radius $R$ revolving around the Sun. The body absorbs the incident solar radiation and it partly reradiates (reflects) the sunlight in the optical band. Directional characteristics of the reflection are described by the so-called bidirectional reflectance function $r$. Given a surface element which is illuminated by the sunlight from the local direction $\boldsymbol{n}_{0}$ (characterized by the zenith angle $\left.\vartheta_{0} ; \mu_{0}=\cos \vartheta_{0}\right)$, the reflectance function yields the radiation (specific) intensity $I$ of the reflected sunlight at the local direction $\boldsymbol{n}$ (charaterized by the zenith angle $\vartheta ; \mu=\cos \vartheta$ ) by

$I\left(\boldsymbol{n} ; \boldsymbol{n}_{0}\right)=I\left(\mu, \mu_{0} ; g\right)=J r\left(\mu, \mu_{0} ; g\right)$.

Here, $\cos g=\boldsymbol{n} \cdot \boldsymbol{n}_{0}$ and $J$ means the radiation intensity of the incident sunlight. The reflectance function $r$ depends on the surface material properties and its roughness. The classical formulations of $r\left(\mu, \mu_{0} ; g\right)$ by Hapke and LummeBowell are reviewed in Bowell et al. (1989).

The thermally reprocessed part of the absorbed energy by the chosen surface element is given by

$\mathcal{E}=J \mu_{0}\left[1-A_{\mathrm{H}}\left(\mu_{0}\right)\right]$,

for $\mu_{0} \geq 0$ and vanishes for $\mu_{0} \leq 0$. Here, $A_{\mathrm{H}}\left(\mu_{0}\right)$ means the hemispheric albedo given by

$A_{\mathrm{H}}\left(\mu_{0}\right)=\frac{1}{J \mu_{0}} \int_{\Omega_{+}} \mathrm{d} \Omega \mu I\left(\mu, \mu_{0} ; g\right)$

( $\Omega_{+}$denotes integration over upper hemisphere). Treatment of the Yarkovsky effect profits from possibility to represent the absorbed energy (2) in terms of the spherical function series

$\mathcal{E}=J \sum_{n \geq 0} \sum_{k=-n}^{n} a_{n k} Y_{n k}(\theta, \phi)$, from which the monopole and dipole terms are relevant in the linearized problem. Here, the spherical angle $\theta$ is measured from the rotation axis of the body and the "longitude" angle $\phi$ from an arbitrary origin in the equatorial plane of the body. A brief algebra yields

$$
\begin{aligned}
& a_{00}=\left(1-A_{1}\right) \frac{\sqrt{\pi}}{2} \\
& a_{10}=\left(1-A_{2}\right) \sqrt{\frac{\pi}{3}} \cos \theta_{0} \\
& a_{1 \pm 1}=\mp\left(1-A_{2}\right) \sqrt{\frac{\pi}{6}} \sin \theta_{0} \mathrm{e}^{\mp i \phi_{0}}
\end{aligned}
$$

with $\left(\theta_{0}, \phi_{0}\right)$ being the solar direction angles in the chosen system of coordinates. As it can be noticed from (5)-(7), the coefficients at each multipole level in the energy development (4) require proper definition of the "albedo" parameter. In general, the necessary albedo parameters are related to the algebraic moments of the hemispheric albedo $A_{\mathrm{H}}\left(\mu_{0}\right)$. Notably, we have defined for each $n \geq 1$

$A_{\mathrm{n}}=(n+1) \int_{0}^{1} \mathrm{~d} \mu_{0} \mu_{0}^{n} A_{\mathrm{H}}\left(\mu_{0}\right)$.

The lowest-degree albedo parameter $-A_{1}-$ coincides with the Bond albedo. Since the monopole energy term (5) determines the mean and subsolar temperatures of the body, the Bond albedo $A_{1}$ affects these values. However, the Yarkovsky thermal force is more intimately linked to the dipole level coefficients (6)-(7) of the energy development (4) and thus more naturally contains the higherdegree albedo coefficient $A_{2}$. The aim of this paper is to show that both the Bond albedo $A_{1}$ and the seconddegree albedo parameter $A_{2}$ appear in evaluation of the Yarkovsky force. However, out of these two parameters, the $A_{2}$ plays a more important role.

The linearized theories of the Yarkovsky force (Rubincam 1995, 1998; Vokrouhlický 1998a,b, 1999) have the same underlying structure (though the proper notation may slightly differ): (i) define the scaled, nondimensional quantities of the problem, (ii) deal with development of the surface irradiation function (4), (iii) solve the linearized heat-diffusion problem, and (iv) compute the thermal (Yarkovsky) force from the dipole temperature distribution on the body. We shall not repeat these steps but only comment where albedo definition plays a role in the theory.

First, the albedo concept contributes to the definition of the scaling temperature (e.g. the subsolar temperature $T_{\star}$ in the notation of Vokrouhlický 1998a, 1999). From (5) we observe that the first-degree moment (Bond) albedo $A_{1}$ is to be used here, so that: $\epsilon \sigma T_{\star}^{4}=\left(1-A_{1}\right) J(\epsilon$ is the IR emissivity and $\sigma$ the Stefan-Boltzmann constant) is a more precise form of Eq. (5) in Vokrouhlický (1998a). Similarly, Eq. (4) of Rubincam (1995) should read: $4 \varepsilon \sigma T_{0}^{4}=\left(1-A_{1}\right) F_{S}^{0}$. The subsolar temperature $T_{\star}$, or the mean temperature $T_{0}$, then provide the important 
factor, tuning the magnitude of the Yarkovsky force, notably the thermal parameter $\Theta$ (or $\Phi$ in the Rubincam's notation; see, e.g., Eq. (6) in Vokrouhlický 1998a, Eq. (4) in Vokrouhlický 1999 or Eq. (11) in Rubincam 1995).

Second, we have seen that the higher-degree albedo coefficients appear in the dipole part of the irradiation function development (4). After including these terms in the boundary conditions of the heat diffusion problem, solving properly the temperature field in the body and evaluating the Yarkovsky force - steps (iii) and (iv) above - we realize that results obtained previously by Rubincam $(1995,1998)$ and those by Vokrouhlický (1998a,b, 1999), Vokrouhlický \& Brož (1999) hold, provided the absorptivity factor that scales all components of the thermal force is interpreted as $1-A_{2}$ (e.g. in the Vokrouhlický's notation $\alpha=1-A_{2}$ and in Rubincam's notation $A=A_{2}$ ).

To summarize the results of this section, we note that the previous linearized solutions for the Yarkovsky force are valid provided the "albedo" factor is properly used. Specifically:

- the Bond albedo $A_{1}$ is used for definition of the scaling temperature and, thus, in evaluating the thermal parameter, and

- the higher-degree albedo $A_{2}$ is used in the scaling factor " $(1-A)$ " in the components of the Yarkovsky force.

Both values $A_{1}$ and $A_{2}$ are numerically close to each other, so that in a very rough zero-order approximation the Bond albedo $A_{1}$ may replace $A_{2}$ in the formulae for the thermal force components. Their difference is briefly discussed below.

\section{Examples and discussion}

Vokrouhlický (1998a,b, 1999) used Lambert reflection law for the incident sunlight. In this highly simplified case, we have $r=a \mu_{0} / \pi$, and one easily shows that $A_{\mathrm{H}}=A_{1}=$ $A_{2}=a$. The surfaces of small bodies in the solar system (e.g., asteroids, satellites), however, do not provide a good fit to Lambert's reflection law.

A more involved representation of the reflection mechanism is that by isotropic scatterers, each charaterized by the single-scattering albedo $w$. If mutual shadowing of the particles is neglected and the surface is assumed perfectly flat, Chandrasekhar (1960) obtained

$$
r\left(\mu, \mu_{0}\right)=\frac{w}{4 \pi} \frac{\mu_{0}}{\mu+\mu_{0}} H(\mu) H\left(\mu_{0}\right),
$$

where the $H$-functions satisfy an integral equation. As has been pointed out by Hapke (1981), a good approximation of its solution is given by $H(x)=(1+2 x) /(1+2 \gamma x)$ with $\gamma=\sqrt{1-w}$ (see also Ahmad \& Deering 1992). The hemispheric albedo is then given by

$A_{\mathrm{H}}\left(\mu_{0}\right)=1-\gamma H\left(\mu_{0}\right)$

and its moments then read

$A_{\mathrm{n}}=1-(n+1) \gamma \int_{0}^{1} \mathrm{~d} \mu \mu^{n} H(\mu)$.

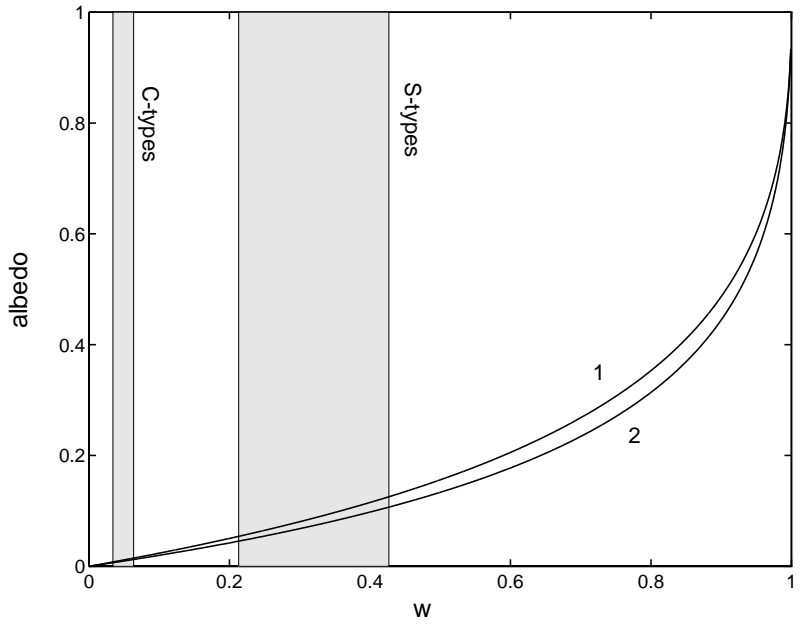

Fig. 1. The first two moment-integrals $A_{1}$ (curve 1) and $A_{2}$ (curve 2) of the hemispheric albedo vs. the single-scattering albedo $w$. The body's surface is represented by the isotropic scatterers. The shaded areas show the characteristic interval of the $w$ parameter for the $\mathrm{S}$ - and $\mathrm{C}$-type asteroids

In the particular case of the isotropic scatterers, Chandrasekhar (1960) has shown an exact relation (see Eq. (18) of Chap. 5 in Chandrasekhar 1960)

$A_{2}=\frac{3}{16} \frac{w}{\gamma^{2}}\left(1-A_{1}\right)^{2}$.

Assuming the above-mentioned Hapke's approximation for the Chandrasekhar's $H$-functions one obtains

$A_{1}=\frac{1-\gamma}{\gamma}\left[1-\frac{\ln (1+2 \gamma)}{2 \gamma}\right]$.

Equations (12) and (13) then yield the necessary information for the Yarkovsky-force evaluation.

Figure 1 shows both albedo-coefficients as a function of the single-scattering albedo $w$. The typical range of this parameter for the $\mathrm{S}$ - and $\mathrm{C}$-type asteroids is shown by the shaded area. We may notice that $A_{2}$ is always a smaller than $A_{1}$, but generally the difference of the two quantities seems very small (quantitatively it is about $17 \%$ of the Bond albedo value for small $w$ and decreases to about $12 \%$ for $w \simeq 0.5$ ).

In a still more complex approximation one may include the effect of anisotropy of the individual scatterers of the surface. Assuming the Henyey-Greenstein phase function with the asymmetry parameter $g$ (see Helfenstein \& Veverka 1989), we may evaluate $A_{1}$ and $A_{2}$ numerically. We have neglected the contribution of backscattering phenomena since they have little effect on the integral quantities such as the hemispheric albedo (e.g. Hapke 1981). Taking into account the asymmetry factor $g$ in the typical interval $(-0.4,0)$, we have numerically computed the first two moments $A_{1}$ and $A_{2}$ of the hemispheric albedo. The results are shown in Fig. 2. We may again conclude a good correspondance between the two albedo-moments (so that $A_{2} \simeq A_{1}$ is still a good zero order approximation), though the $A_{2}$ value is systematically smaller than $A_{1}$. We have 


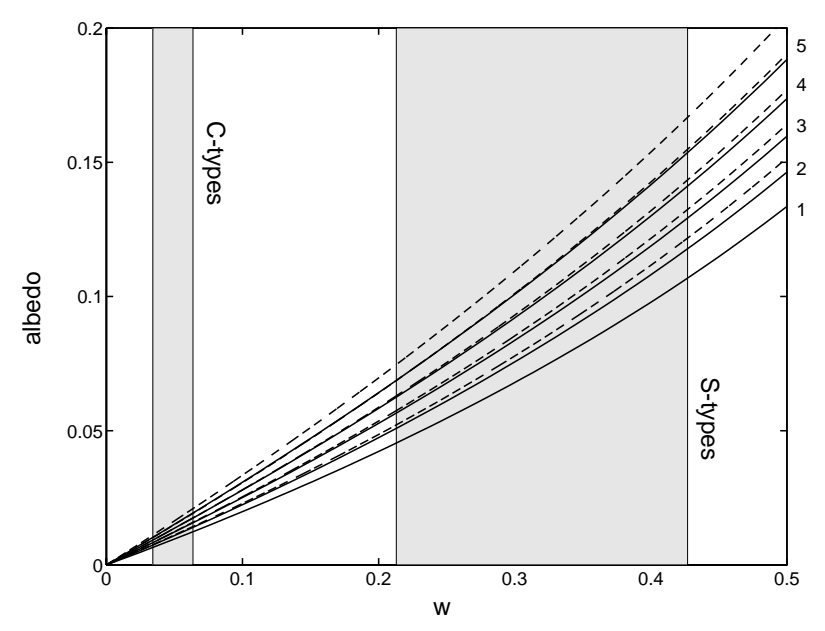

Fig. 2. The first two moment-integrals $A_{1}$ (dashed curves) and $A_{2}$ (solid curves) of the hemispheric albedo vs the singlescattering albedo $w$. Surface represented by the individual scattering particles with a phase function given by the HenyeyGreenstein formula. Four typical values of the asymmetryparameter $g$ assumed: (i) $g=0$ (curves 1), (ii) $g=-0.1$ (curves 2), (iii) $g=-0.2$ (curves 3 ), (iv) $g=-0.3$ (curves 4 ), and (v) $g=-0.4$ (curves 5 )

checked that adding the effects of the surface roughness does not change the conclusions derived from the above examples.

Finally, let us also comment on the correct usage of the albedo parameter in the non-linearized theories of the Yarkovsky effect (e.g. Vokrouhlický \& Farinella 1998, 1999; Spitale \& Greenberg 2001). Such theories solve the heat diffusion problem numerically, typically on a grid that covers spherical coordinates on the body and time. When formulating the (non-linearized) boundary condition of the heat-diffusion problem for the individual surface elements, one should use the hemispheric albedo $A_{\mathrm{H}}$ defined in (3) above. Note in particular that this quantity depends on the zenith angle $\left(\vartheta_{0}\right)$ of the incident sunlight. To our knowledge, none of the numerical approaches have included this effect so far (since they typically assumed $A_{\mathrm{H}} \simeq$ const.), so that investigation of the hemispheric albedo variability with the zenith angle for the numerical evaluation of the Yarkovsky force is left for future work (the hemispheric albedo may change by a factor of $\simeq 2$ between the zenith and horizontal incindent angle of the sunlight).
In this paper, we relaxed constraining assumptions about the surface reflectivity from some previous analyses. Despite this, we have still assumed Lambert (isotropic) emission in the infrared (IR) band. Note that this assumption was implicitly used for formulating the Yarkovsky force. Observations and theory suggest that the directional characteristics of the IR emission are anisotropic to some degree (e.g. Lebofsky \& Spencer 1989; Spencer 1990), but the quantitative modeling of this phenomenon is not as advanced as, say, those in the optical band. We believe the inclusion of anisotropic IR emission into our model might be an interesting (though complicated) project for the future, as might be a study of the possible variation of the reflectance pattern (i.e., the $r$-function) on the body's surface.

Acknowledgements. We thank Paul Helfenstein for several useful discussions of this topic. Support for W.F. Bottke was provided by NASA Grant NAGW-310.

\section{References}

Ahmad, S. P., \& Deering, D. W. 1992, J. Geophys. Res., 97, 18867

Bottke, W. F., Rubincam, D. P., \& Burns, J. A. 2000, Icarus, 145,301

Bowell, E., Hapke, B., Domingue, D., et al. 1989, in Asteroids II, ed. R. P. Binzel, T. Gehrels, \& M. S. Matthews (The University of Arizona Press, Tucson), 524

Chandrasekhar, S. 1960, Radiative Transfer (Dover, New York) Hapke, B. 1981, J. Geophys. Res., 86, 3039

Helfenstein, P., \& Veverka, J. 1989, in Asteroids II, ed. R. P. Binzel, T. Gehrels, \& M. S. Matthews (The University of Arizona Press, Tucson), 557

Helfenstein, P., et al. 1996, Icarus, 120, 48

Lebofsky, L. A., \& Spencer, J. R. 1989, in Asteroids II, ed. R. P. Binzel, T. Gehrels, \& M. S. Matthews (The University of Arizona Press, Tucson), 128

Rubincam, D. P. 1995, J. Geophys. Res., 100, 1585

Rubincam, D. P. 1998, J. Geophys. Res., 103, 1725

Spencer, J. R. 1990, Icarus, 83, 27

Spitale, J., \& Greenberg, R. 2001, Icarus, 149, 222

Vokrouhlický, D. 1998a, A\&A, 335, 1093

Vokrouhlický, D. 1998b, A\&A, 338, 353

Vokrouhlický, D. 1999, A\&A, 344, 362

Vokrouhlický, D., \& Brož, M. 1999, A\&A, 350, 1079

Vokrouhlický, D., \& Farinella, P. 1998, AJ, 116, 2032

Vokrouhlický, D., \& Farinella, P. 1999, AJ, 118, 3049 